\title{
TITLE:
}

\section{Laser ablation at solid-liquid interfaces: An approach from optical emission spectra}

\section{AUTHOR(S):}

Sakka, T; Iwanaga, S; Ogata, YH; Matsunawa, A; Takemoto, $\mathrm{T}$

\section{CITATION:}

Sakka, T ... [et al]. Laser ablation at solid-liquid interfaces: An approach from optical emission spectra. JOURNAL OF CHEMICAL PHYSICS 2000, 112(19): 8645-8653

\section{ISSUE DATE:}

2000-05-15

URL:

http://hdl.handle.net/2433/50406

\section{RIGHT:}

Copyright 2000 American Institute of Physics. This article may be downloaded for personal use only. Any other use requires prior permission of the author and the American Institute of Physics. 


\title{
Laser ablation at solid-liquid interfaces: An approach from optical emission spectra
}

\author{
Tetsuo Sakka, ${ }^{\text {a) }}$ Shuji Iwanaga, and Yukio H. Ogata \\ Institute of Advanced Energy, Kyoto University, Uji, Kyoto 611-0011, Japan \\ Akira Matsunawa and Tadashi Takemoto \\ Joining and Welding Research Institute, Osaka University, Ibaraki, Osaka 567-0047, Japan
}

(Received 14 January 2000; accepted 24 February 2000)

\begin{abstract}
The emission spectra from the solid-liquid interface irradiated by a pulsed laser were studied. The solid target used in this study was graphite and boron nitride, and the liquid in which the target was immersed was water, benzene, $n$-hexane, and carbon tetrachloride. The results showed strong continuous spectrum immediately after a pulse shot, whereas after $\approx 100$ ns later from the irradiation it was greatly reduced, and instead, the emission from small molecules dominated the spectra. The line spectra of small molecules observed in the later time range indicate the chemical reaction between the ablated species and the species originated from the liquid molecules. The intensity of the continuous spectrum was very prominent compared to what has been observed for solid-gas interfaces. This is due to rapid electron ion recombination or bremsstrahlung due to highly confined interface plasma. (C) 2000 American Institute of Physics. [S0021-9606(00)70619-2]
\end{abstract}

\section{INTRODUCTION}

Pulsed laser ablation is a method often used for obtaining atoms, ions and small molecules from solid materials. ${ }^{1-3}$ This technique enables even the evaporation of refractory metals and ceramics, because the energy density sufficient for the evaporation is easily attained at the irradiation spot. Some of the ablation species are electronically excited, and are expected to be very reactive. Many researchers have investigated laser ablation in vacuum or in diluted gases, aiming at thin film fabrication and cluster formation. ${ }^{1-3}$ These studies revealed that the reaction between ablation species and the gas molecules pre-introduced in the sample chamber is possible ${ }^{4-6}$ and gives important species for laser material processing.

On the other hand only a few studies have been reported for the laser ablation at solid-liquid interfaces, ${ }^{7}$ probably because of their comparatively limited application from the materials processing view point. However, if the ablation species have a chance to encounter the molecules in the liquid phase without being deactivated, various chemical reactions between the ablation species and the molecules in the liquid phase are expected, since the most nascent ablation species are highly excited both in electronically and in translationally.

When a solid target is submerged in a transparent liquid, the initial process of the laser ablation is an interaction of the light with the solid target surface. This means that the state of the ablation species at the very initial stage should be independent from the liquid contacting the solid target, and hence we can expect that such ablation species as obtained in the vacuum have a chance to encounter the molecules in liquid covering the surface. Therefore, by investigating this

${ }^{a)}$ Author to whom correspondence should be addressed. process we may possibly be able to clarify dynamic properties of excited species in liquid, which is an important subject in the field of chemical reaction. Since the publication in this field is very limited, we first attempted, in the present study, to clarify the behavior of the ablation species during the pulsed laser irradiation to solid-liquid interfaces.

Several studies have been reported on the pulsed laser irradiation onto solid-liquid interfaces. ${ }^{7-11}$ A series of studies have been devoted to laser peening for the purpose of materials surface processing. ${ }^{8-10}$ This technique utilizes the instantaneous increase in local pressure produced at the irradiation spot. The pulsed laser irradiation has also been applied to solid particles suspended in liquid. Spectroscopic study has shown a spatial anisotropy in spectral behavior. ${ }^{12}$ Also it has been pointed out that the particle size is reduced by the irradiation, ${ }^{13}$ which suggests the emission of some kind of species from the solid surface into the liquid. One of the very few studies performed for the purpose of clarifying the mechanism of the laser ablation of solid materials submerged in liquid is that by Ageev et al. ${ }^{7}$ They investigated gas cavity formation by the particles ablated from the solid surface into liquid, and shock wave propagation in the liquid. The other work, we know, published on the laser ablation at the solid-liquid interface is that by Wakisaka et al., ${ }^{11}$ in which product analysis of the liquid phase was performed. By these preceding works it has been clarified that pressure of the irradiated spot becomes extremely high, that the ablation species will impinge into the liquid phase, and that the chemical reaction between ablated species and the liquid molecules can occur. For the further pursuit of the reaction between ablation species and liquid molecules, timedependent analysis of the species present at the interface will provide important information, i.e., the knowledge on the lifetime of the ablation species will help us consider the way how the chemical reaction occurs in the excited region pro- 


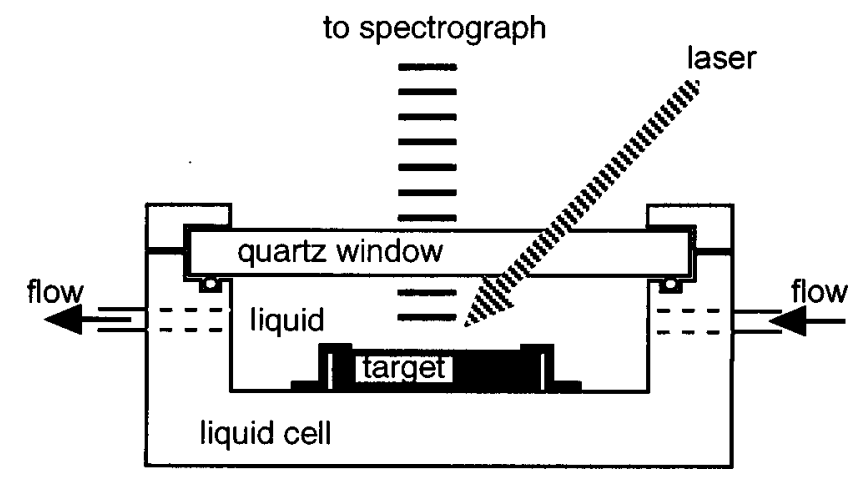

FIG. 1. Schematic illustration of the irradiation cell.

duced by the laser ablation at the solid-liquid interface.

In this paper we demonstrate time resolved optical emission spectra of the laser ablation plasma at solid-liquid interfaces. Here we show a spectral evidence of molecular formation from the species ablated from the solid surface submerged in liquid.

As a target material we have chosen graphite and boron nitride. Laser ablation of these materials in vacuum or in diluted gases has been extensively studied., ${ }^{4,511,14-23}$ Since various carbon species have been observed by using graphite as a target, the investigation of the laser ablation of graphite in liquid would give important information for organic chemistry of reactive carbons. Since boron nitride is a compound with the electronic structure similar to graphite, we chose this material for another target material. Water, benzene, $n$-hexane, and carbon tetrachloride were employed as the liquids.

\section{EXPERIMENT}

The $1064 \mathrm{~nm}$ wavelength radiation of the Nd:YAG laser with the pulse width of $20 \mathrm{~ns}$ was focused on the solid target immersed in a liquid. The energy fluence of the pulse was $\approx 8-9 \mathrm{~J} / \mathrm{cm}^{2}$. An illustration of the irradiation cell is given in Fig. 1. By the irradiation condition employed here, the breakdown does not occur without a solid target. The liquid in the cell was circulated and filtered continuously by a pump to renew the liquid covering the target. In this way the absorption of the laser radiation by the species produced by the preceding pulses was avoided. Time resolved spectra of the surface plasma emission were obtained by using a $27 \mathrm{~cm}$ focal length spectrograph (Acton Research, Inc., SpectraPro275) and an intensified charge coupled device (ICCD) (Princeton Instruments, Inc., ICCD-1024MTDGE/1) as a detector. The active area of the CCD surface was $\sim 900 \times 256$ pixels. The emission from the plasma was collected by a lens system and focused into an entrance slit of the spectrograph. The width of the slit was adjusted to $20 \mu \mathrm{m}$. A diffraction grating with 300 grooves $/ \mathrm{mm}$ was used to record a wide range of wavelength simultaneously. The ICCD exposure was controlled by imposing a high voltage pulse generated by a gate pulser (Princeton Instruments, Inc., PG-200) to the intensifier. The duration of the gate was set to be $15 \mathrm{~ns}$ and the time delay from the laser pulse was varied within the range from 29 ns to 2000 ns. For each spectrum signal was accumulated to obtain good signal to noise ratio.

Because of a short exposure time and the cooling to $-30{ }^{\circ} \mathrm{C}$ the dark count of the $\mathrm{CCD}$ detector was negligibly small. However, the sensitivity of the detector and the throughput of the spectrograph depend on the wavelength, and furthermore, the edge of the detector is less sensitive than the center because of the fast pulse drive of the ICCD as well as the intensifier nature. Those were corrected by the following manner. The spectral sensitivity of the detector and the throughput of the spectrograph, which can be approximated by the reflectance of the grating, were corrected by the use of the data provided from the manufacturer. The sensitivity decrease in the edge region of the ICCD surface driven by the $15 \mathrm{~ns}$ intensifying pulse was estimated by comparing the signal intensities of a certain wavelength portion of a high pressure Xe lamp measured by various parts of the ICCD surface by rotating the grating. By using this sensitivity factor, the intensity observed in the edge region was corrected.

Graphite used was a polycrystalline isotropic block, of which the purity was $99.99 \%$. Boron nitride of polycrystalline tablet was purchased from Kojundo Chemical Laboratory Co., Inc. The purity was $99 \%$. All the liquid chemicals employed in the present study were used as purchased without further purification. The water used in the experiments was a distilled water purchased from Nacalai Tesque, Inc. Benzene and $n$-hexane were spectral grade purchased from Nacalai Tesque, Inc. and the purity was $99.7 \%$ and $97 \%$, respectively. Carbon tetrachloride was fluorometry grade purchased from Kanto Chemical Co., Inc., and the purity was $99.7 \%$.

\section{RESULTS}

In Fig. 2 the spectra obtained by irradiating the graphite target submerged in water are shown. Each spectrum in Fig. 2 represents a different delay time from the laser pulse. In each figure the data accumulation number is shown. Broken lines in the figures denote the best fit to Planck's formula for the black body radiation. A broad or continuous spectrum dominated the emission spectrum up to $\approx 100$ ns of the delay time, while at later times the continuous emission was greatly reduced and the series of emission lines became dominant in the spectra. The intensity of the spectrum depends strongly on the pulse energy fluence, and also sensitive to the surface state of the target, i.e., a small fluctuation of the laser pulse results in a large difference in the intensity of the emission. This means that the intensity of the spectra cannot be compared quantitatively. However, the tendency seen in the spectra that the broad spectrum faded out in $\approx 100 \mathrm{~ns}$, and that the whole spectrum disappears in a couple of microseconds would be qualitatively correct. It should be noted that each spectrum is a result of integrating the emission signal of the different number of the ablation events, of which the number is stated in each figure. For example, the spectra of the delay time from $29 \mathrm{~ns}$ to $134 \mathrm{~ns}$ were the results of the integration of 5 shots of pulses, while those of the delay time from $400 \mathrm{~ns}$ to $2000 \mathrm{~ns}$ were the results of the integration of 10 shots. 


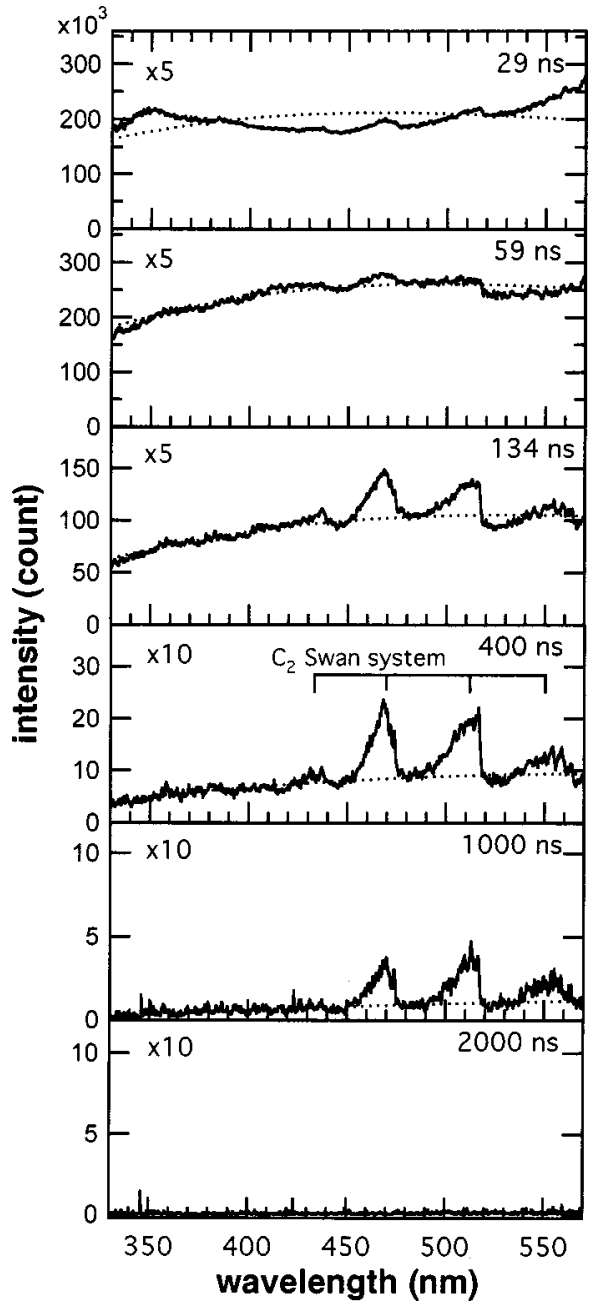

FIG. 2. Emission spectra from the graphite-water interface irradiated with a pulsed Nd:YAG laser. Broken line is a curve fitting by the modified Planck formula, Eq. (A1). The delay time from the laser irradiation is given in each spectrum. The number indicated in the upper left of each spectrum is the pulse accumulation number for the data acquisition.

In Figs. 3-5 the results for the emission spectra, when benzene, $n$-hexane, carbon tetrachloride were used as the liquid, respectively, are shown. Each figure has 6 spectra representing different delay time from the pulse shot. The general feature of the spectra is very similar to that in the case of water. However, in the cases of benzene and carbon tetrachloride fairly prominent line spectra can be distinguished even at very early stage of the emission.

In Figs. 6-9 the results for boron nitride are shown. Figure 6 shows the emission spectra from boron nitride submerged in water. In the early stage of the emission, the spectra were dominated by continuous spectrum. In the case of the delay time of $29 \mathrm{~ns}$ or $44 \mathrm{~ns}$, the wavelength dependence of the continuous spectrum is obviously different from that of black-body radiation. The only line spectrum observed is that at $346 \mathrm{~nm}$. In contrast to the case of graphite the spectra do not show any emission line in the wavelength range from 400 to $550 \mathrm{~nm}$ until $200 \mathrm{~ns}$ after the pulse. Later a progression of line spectra appears, as can be clearly seen in the spectrum of the delay time of $434 \mathrm{~ns}$. This structure eventually disappeared after a couple of microseconds from the

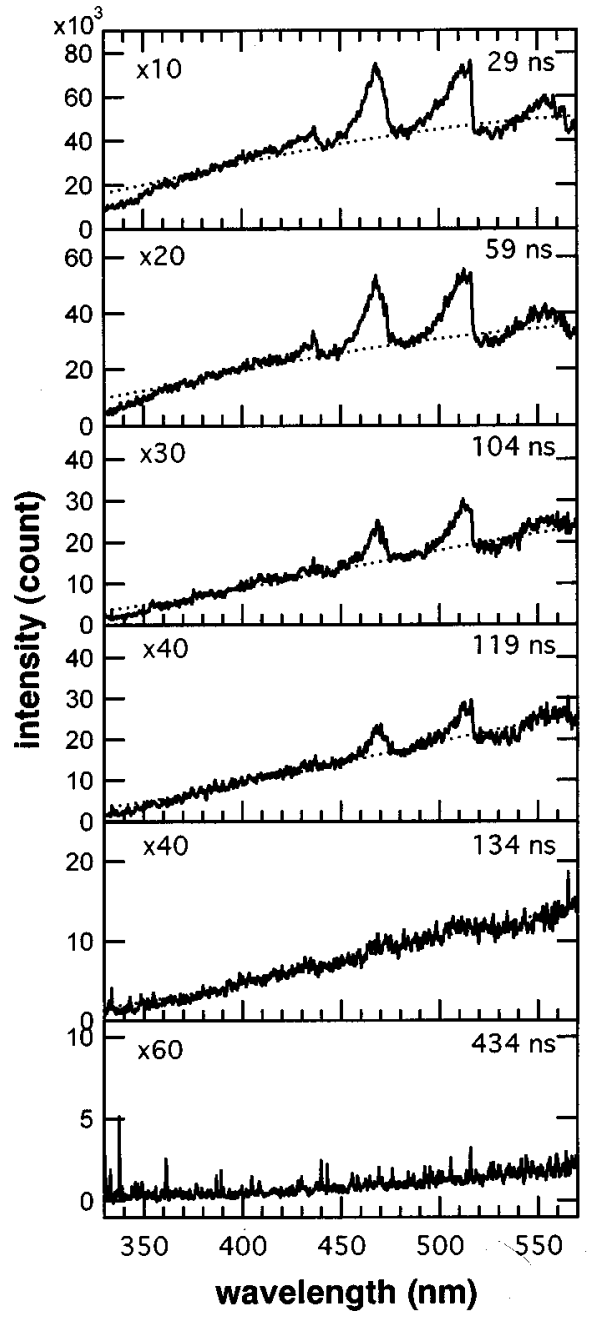

FIG. 3. Same as Fig. 2 except that the emission spectra from the graphitebenzene interface is recorded in this figure.

pulse irradiation. Figure 7 shows the results for boron nitride submerged in benzene. The spectra in the early stage of the emission show a prominent line spectrum at $346 \mathrm{~nm}$, as in the case of boron nitride in water. However, the spectrum in the range from $450 \mathrm{~nm}$ to $550 \mathrm{~nm}$ shows the feature similar to that of graphite. The spectra shown in Fig. 8 indicate very large continuous spectrum, especially in an early stage. However, the observed line spectra are quite similar to that of Fig. 7. Figure 9 shows the spectra obtained from the boron nitride-carbon tetrachloride system. This is again very similar to Fig. 8, except that in these spectra the sharp peak at $346 \mathrm{~nm}$ is absent even in a very early spectrum.

\section{DISCUSSION}

The time dependence of the emission spectra observed here is expected to give us important information on the reaction process. However, the chemical species detected by the emission spectra are limited to the species which have an emission line in the observed spectral range. It should be always kept in mind that we are looking at a part of the species in the whole system. At the same time it is also the reality that very few methods are existent which could clarify the time evolution of the chemical species in the laser in- 


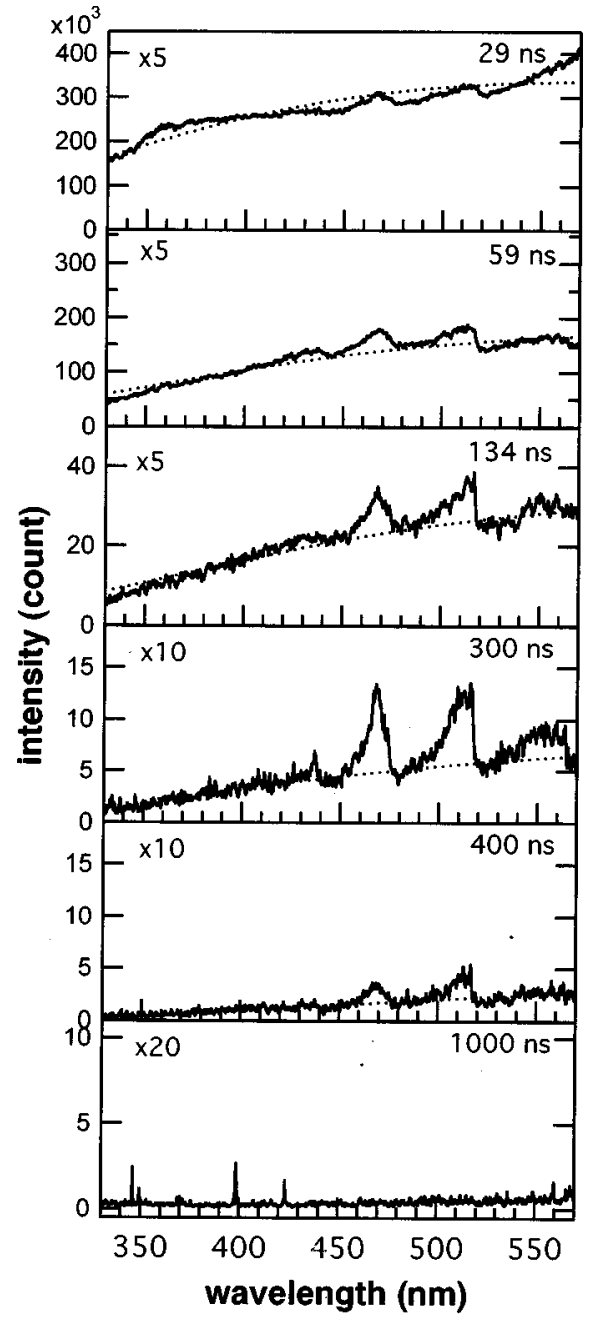

FIG. 4. Same as Fig. 2 except that the emission spectra from the graphite$n$-hexane interface are shown in this figure.

duced plasma at liquid-solid interfaces. Therefore, the effort here should be recognized as one attempt to clarify time evolution of the ablation species, even though the observation is limited to a part of the ablation species.

All the solid-liquid systems investigated in the present study gave results showing an intense continuous spectra in the early stage of the plasma emission. After several hundreds of nanosecond clear line spectra were seen for all the cases. The assignment of the line emission spectra is listed in Table I. It is based on spectral line handbooks ${ }^{24-27}$ and some literatures. ${ }^{4,11}$ Most spectral lines observed here are assigned to electronic transitions of diatomic molecules and their vibrational progressions, i.e., $\mathrm{C}_{2}$ Swan band, $\mathrm{CN}$ violet band, and $\mathrm{BO} \alpha$ band. Contrary to the optical emission spectra observed in vacuum the line spectra due to ions and neutral atoms are greatly suppressed. The only ion line spectrum observed in this study was that at $346 \mathrm{~nm}$, which was assigned to a radiative transition of boron ion. However, it should be noted that the above results of the assignment do not necessarily mean the absence of the ions in the nascent laser-induced plasma. We rather think that the emission from the ions was suppressed by fast electron-ion recombination which gives intense continuous spectra. This is discussed in

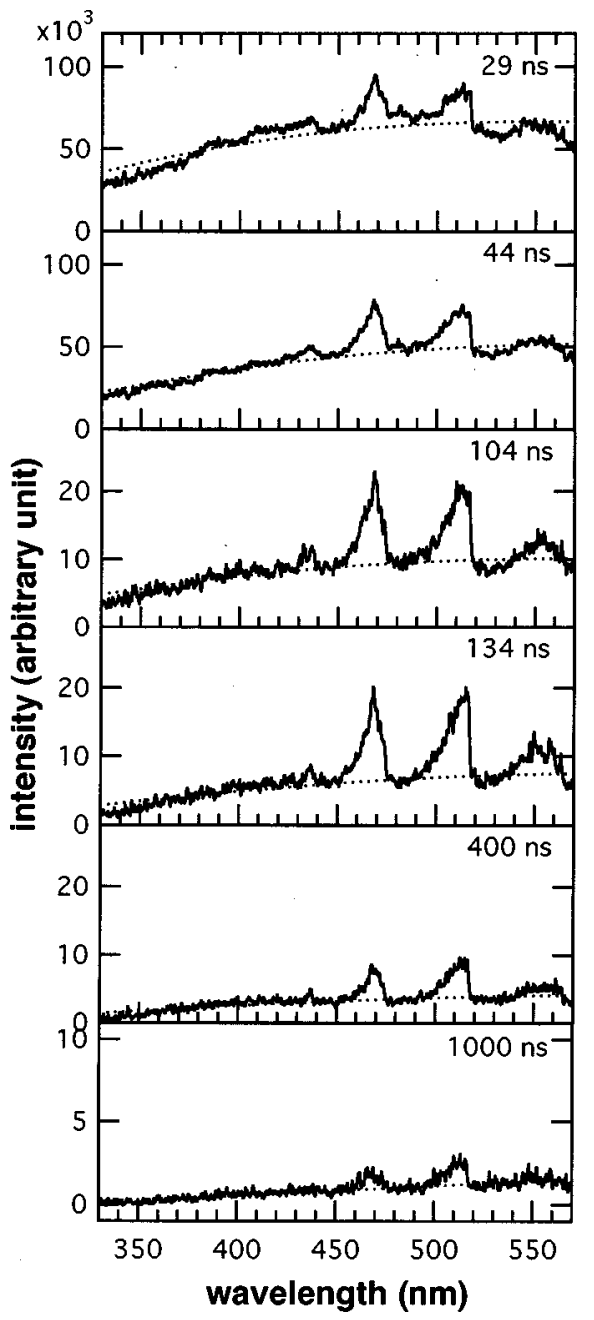

FIG. 5. Same as Fig. 2 except that the emission spectra from the graphitecarbon tetrachloride interface are shown in this figure.

the following paragraph and also in the Appendix, in detail, in relation to the mechanism of the appearance of the continuous spectrum.

Generally, there are several possibilities to cause continuous spectra, namely, bremsstrahlung, radiative electronion recombination, the Stark broadening, black body radiation, etc. ${ }^{28-34}$ The clarification of the origin of the continuous spectra will bring out some information on the reaction dynamics at the solid-liquid interface at which the pulsed-laser is irradiated. Although the origin of the broad continuous spectrum is not clear up to now, it is probably due to bremsstrahlung and/or electron-ion recombination in the plasma, and the spectra of the larger delay time may be explained partly by a blackbody radiation of the solid surface. Since the discussion on the mechanism of the appearance of the continuous spectra is not the major subject of this paper, it is described in the Appendix in some detail.

In the spectra of boron nitride-water system (Fig. 6) the presence of $\mathrm{BO}$ molecules becomes apparent at $\approx 400 \mathrm{~ns}$ after the irradiation. Since there is no oxygen source in the boron nitride crystal, the oxygen atom in this molecule must have originated in water. Also, in the spectra for boron nitride in benzene (Fig. 7), $n$-hexane (Fig. 8), and $\mathrm{CCl}_{4}$ (Fig. 


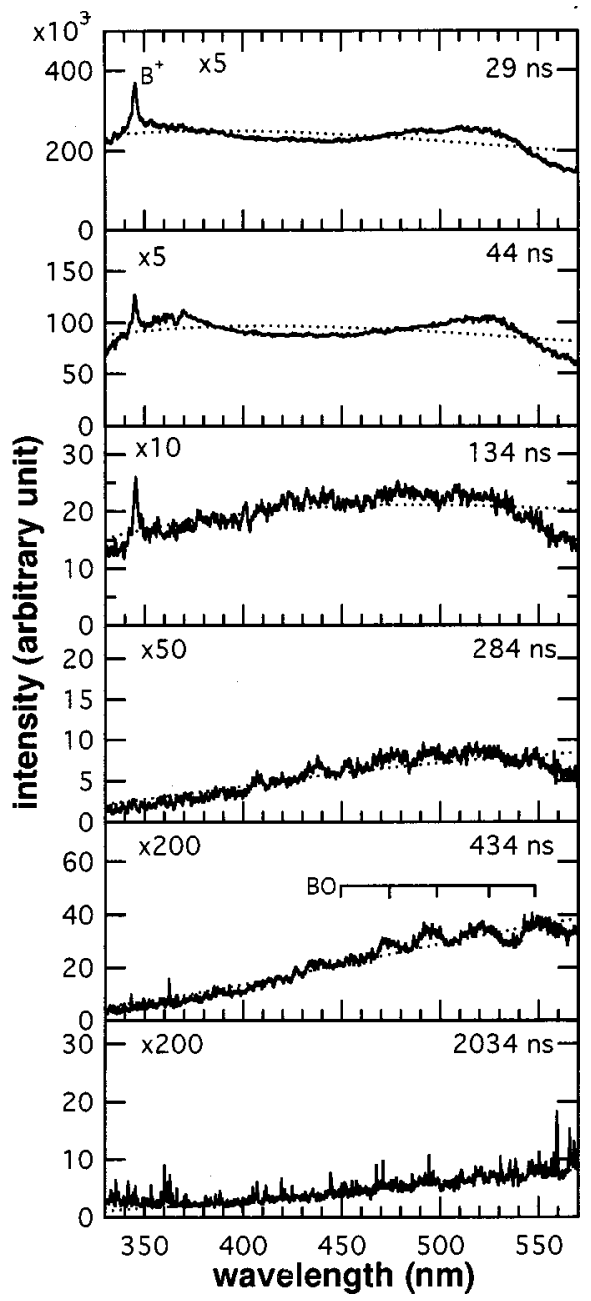

FIG. 6. Same as Fig. 2 except that the emission spectra from the boron nitride-water interface are shown in this figure.

9), the presence of $\mathrm{C}_{2}$ and $\mathrm{CN}$ is shown. Similarly, the carbon atom in $\mathrm{C}_{2}$ and $\mathrm{CN}$ should have originated in liquid molecules. The presence of $\mathrm{BO}$ or $\mathrm{CN}$ molecules is evidence of the reaction between the species ablated from the solid surface and the species originated in the liquid.

The reaction of the boron atom with the water molecule in gaseous phase has been studied by Andrews and Burkholder. ${ }^{6}$ It has been shown that the production of $\mathrm{BO}$ is a major channel for the gas phase reaction. For the case of the reaction in liquid the liquid molecules are most probably involved in the laser-induced plasma, somehow, during the laser irradiation. Since the laser intensity employed in the present study does not result in the breakdown of the liquid in the absence of a solid target, the very initial stage of the excitation of the interfacial region must be the process initiated by the ablation of the solid target. However, it is possible that the highly excited ablation species penetrate into the liquid and change the liquid properties related to the absorption of the laser energy, or directly react with the molecules in the liquid to form a hot region where the emission of the visible light is possible. These processes will result in further absorption of a later part of the laser pulse, which excites the interfacial region more strongly to form a plasma. This mechanism explains very well the incorporation of the

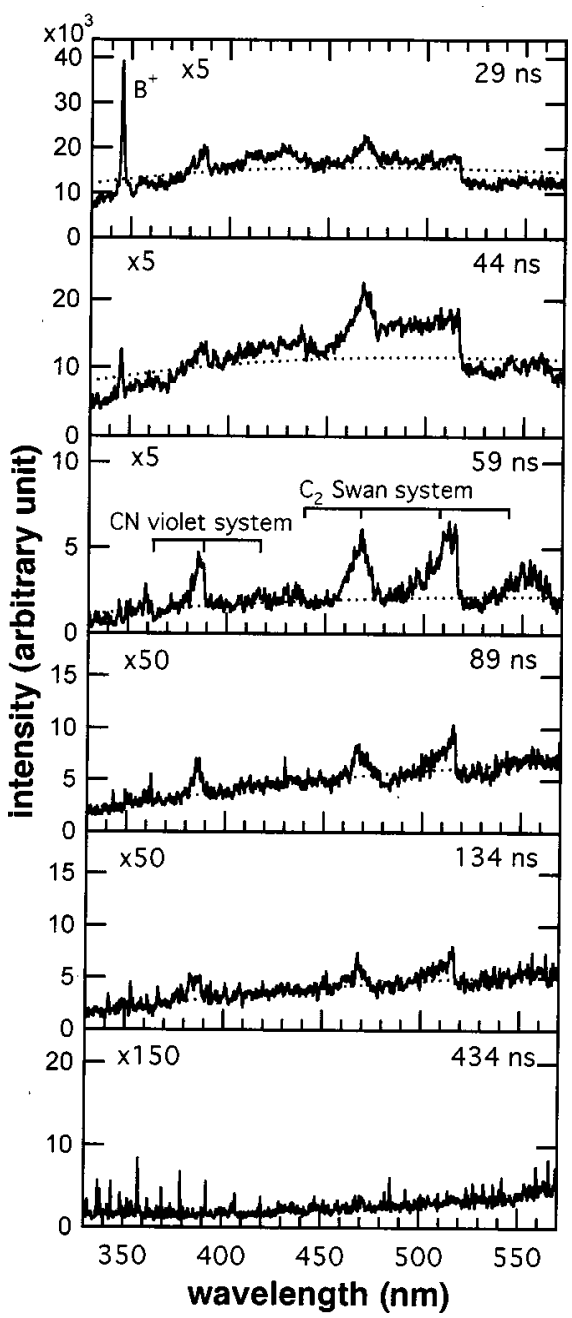

FIG. 7. Same as Fig. 2 except that the emission spectra from the boron nitride-benzene interface are shown in this figure.

atoms originally in liquid molecules into the plasma. The effects of the initial ablation species upon the absorbance of the liquid at the interface region can be clarified by the use of ultrafast lasers, i.e., if the pulse width is shorter than the time scale expected for the change in the absorbance of the liquid, the extra excitation by the later part of the laser pulse would be avoided. Unfortunately, the experiment using a femtosecond laser source for an excitation of a solid-liquid interface has not been performed so far.

Our results show that in the early stage the emission spectra are dominated by the continuous spectra. If the continuous spectra are due to the radiative electron-ion recombination or the bremsstrahlung, the plasma density is roughly estimated to be $\sim 10^{20} \mathrm{~cm}^{-3}$ (see Appendix). Although the characteristic ion emission lines are not observed in the spectra, this suggests that there should have been many ions in the interface laser-induced plasma. This is pretty well consistent with the following discussion on the product analysis in the case of graphite or boron nitride in benzene.

Product analysis was performed to ensure the further reaction between the species observed by the spectra and liquid molecules. This experiment was performed for the graphitebenzene and boron nitride-benzene systems. Gas chromato- 


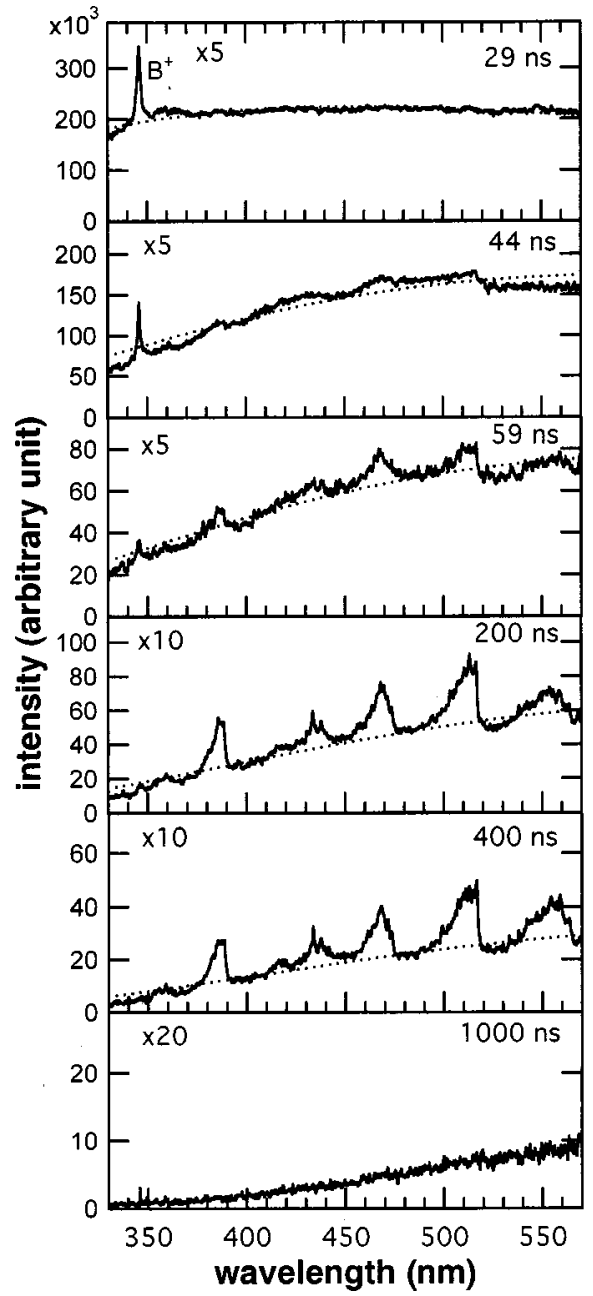

FIG. 8. Same as Fig. 2 except that the emission spectra from the boron nitride- $n$-hexane interface are shown in this figure.

graph mass spectroscopic analysis was employed for the liquid after the irradiation of several thousands of laser shots. The results are listed in Table II. Two products were observed in the analysis, toluene, and phenylacetylene. These products correspond to the reaction of the $\mathrm{C}$ atom or ion $\left(\mathrm{C}_{1}\right.$ species, hereafter) and $\mathrm{C}_{2}$, respectively, with benzene molecules. The results are consistent with the results of Wakisaka et al., ${ }^{11}$ where the series of products, including the products observed in the present study, were reported. However, our spectra do not show explicitly the presence of $\mathrm{C}_{1}$ species (Figs. 2-9), contrary to the case of the laser ablation of graphite in vacuum or in diluted gases. It is most likely that $\mathrm{C}_{1}$ species originally produced in the surface plasma relax into $\mathrm{C}_{2}$ or react with molecules in liquid very rapidly, and further, the emission from these species is obscured by the very intense emission due to the electron-ion recombination. The results in Table II show that the relative yield of toluene and phenylacetylene is independent from the target type. If both $\mathrm{C}_{1}$ species and $\mathrm{C}_{2}$ have been produced directly from graphite or benzene, the relative yield of $\mathrm{C}_{1}$ species and $\mathrm{C}_{2}$ can be different for different carbon sources, and hence, the relative yield of toluene and phenylacetylene should depend on the carbon source. Recalling that $\mathrm{C}_{2}$ is observed even for the case of graphite in water (Fig. 2), graphite cer-

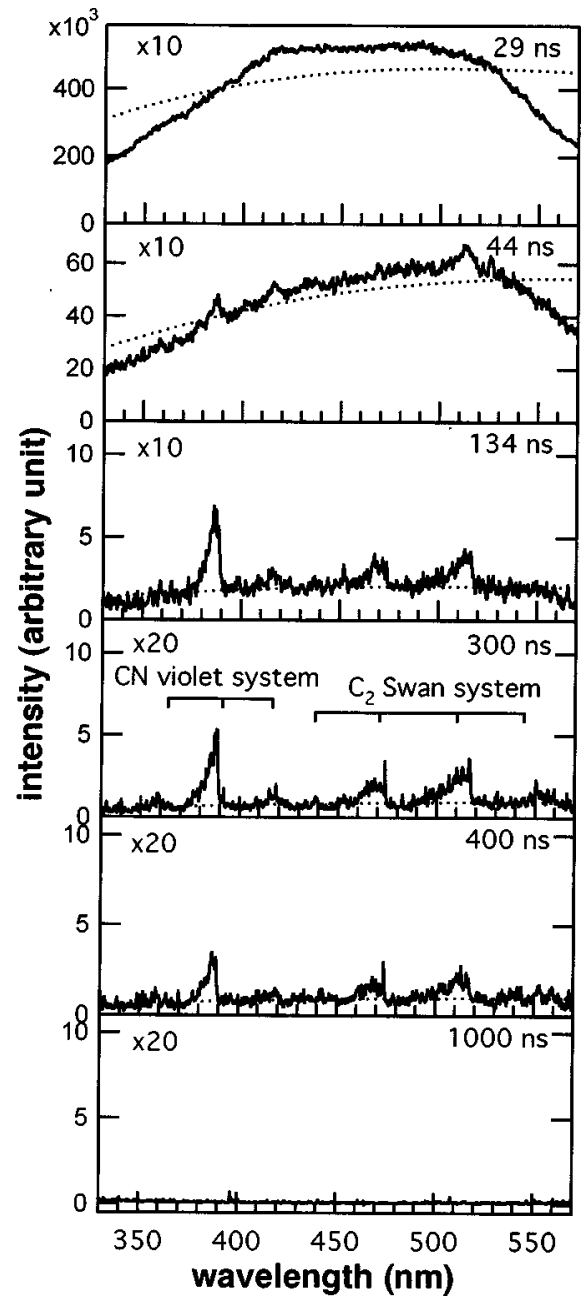

FIG. 9. Same as Fig. 2 except that the emission spectra from the boron nitride-carbon tetrachloride interface are shown in this figure.

tainly emits carbon species. This means that the carbon source for the graphite-benzene system is both benzene and graphite while that of the boron nitride-benzene system is only benzene. In spite of this difference the results showed the same relative yield of toluene and phenylacetylene. This is consistently explained, if we assume that the original carbon species produced by the laser irradiation are all $\mathrm{C}_{1}$ spe-

TABLE I. Assignment of the emission lines.

\begin{tabular}{clc}
\hline \hline Wavelength $(\mathrm{nm})$ & \multicolumn{1}{c}{ Species } & Transition \\
\hline 359.0 & $\mathrm{CN}(\Delta \nu=+1)$ & \\
385.5 & $\mathrm{CN}(\Delta \nu=0)$ & $B{ }^{2} \Sigma^{+}-X^{2} \Sigma^{+}$ \\
418.5 & $\mathrm{CN}(\Delta \nu=-1)$ & \\
436.7 & $\mathrm{C}_{2}(\Delta \nu=+2)$ & \\
468.2 & $\mathrm{C}_{2}(\Delta \nu=+1)$ & $d^{3} \Pi_{g}-a^{3} \Pi_{u}$ \\
516.1 & $\mathrm{C}_{2}(\Delta \nu=0)$ & \\
563.3 & $\mathrm{C}_{2}(\Delta \nu=-1)$ & \\
345.1 & $\mathrm{~B}^{+}$ & \\
435.5 & $\mathrm{BO} 2 p^{1} \mathrm{P}^{0}-2 p^{2}{ }^{1} \mathrm{D}$ \\
470.8 & $\mathrm{BO}$ & \\
490.5 & $\mathrm{BO}$ & \\
521.3 & $\mathrm{BO}$ & \\
548.3 & $\mathrm{BO}$ & \\
\hline \hline
\end{tabular}


TABLE II. Relative yield of the reaction products in the case of graphite and boron nitride in benzene.

\begin{tabular}{lclc}
\hline \hline Target & $\begin{array}{c}\text { Fluence } \\
\left(\mathrm{J} \mathrm{cm}^{-2}\right)\end{array}$ & Reaction products & Relative yield \\
\hline $\begin{array}{l}\text { no target } \\
\text { graphite }\end{array}$ & 6.0 & $\begin{array}{l}\text { no product } \\
\text { toluene } \\
\text { phenylacetylene }\end{array}$ & 1 \\
toluene & 1.1 \\
graphite & 6.0 & 0.5 & $\begin{array}{l}\text { phenylacetylene } \\
\text { toluene } \\
\text { phenylacetylene }\end{array}$ \\
boron nitride & 6.0 & 0.9 \\
& & & 1.1 \\
\hline \hline
\end{tabular}

cies, regardless to the source of carbon, namely, graphite, benzene, or both. The $\mathrm{C}$ ions or atoms produced in this way then dimerize or react with benzene molecules. By assuming a collisional reaction which follows simple kinetics, the branching ratio of dimerization and the reaction with benzene is only a function of the density and temperature. If the density of the initial $\mathrm{C}_{1}$ species and the temperature are the same for different solid-liquid systems, the ratio of the final products should also be the same. Also, according to the simple collision reaction, the rate of the dimerization of $\mathrm{C}_{1}$ species follows the square of the density of the $C_{1}$ species, while the reaction with benzene follows linear to the density of $\mathrm{C}_{1}$ species. Therefore, if the density of $\mathrm{C}_{1}$ species is changed, the ratio of the final products could be changed. This is consistent with the results that the low fluence irradiation gives the lower relative yield of phenylacetylene. Furthermore, the absence of $\mathrm{C}_{2}$ in the nascent plasma is consistent with the time dependence of the emission spectra, where the $\mathrm{C}_{2}$ Swan band appears in later time regime.

\section{CONCLUSION}

The spectroscopic study of the laser ablation at solidliquid interfaces showed that (1) reactions between the species ablated from the solid target and the ambient liquid molecules are evidenced by the emission spectroscopy, (2) not only the species from the solid target but also the species originated from the liquid are involved in the interface plasma, (3) broad backgroundlike spectrum is always observed in the early stage of the emission, suggesting rapid electron ion recombination due to a strong confinement of the interface laser-induced plasma, and (4) the ions can be observed in the early stage of the emission, whereas molecular spectra become dominant in later stages.

The behavior is pretty different from the case of solidgas interfaces. The difference is summarized from the point of view of the density of the laser-induced plasma. Both the appearance of an intense continuous spectra and the obscurity of radiative transition of ions and atoms can be explained by a strong compression of the emission species into a small region at the interface, resulting in an efficient electron-ion recombination giving an intense continuous spectra, and also the rapid decay of atoms and ions.

\section{ACKNOWLEDGMENTS}

We would like to acknowledge Professors Mahito Mabuchi (Niihama National College of Science and Technology), Takashi Nakajima (Kyoto University), and S. L. Chin (Laval University) for their comments on the continuous spectra. Technical support of Kokichi Hotta is also acknowledged. This work is financially supported partly by the Joint Research Program between JWRI, Osaka University, and IAE Kyoto University, and partly by a Grant-in-Aid on Priority Area of "Ordered Interface in Electrochemistry" from the Ministry of Education, Science, Sports, and Culture in Japan.

\section{APPENDIX}

In this Appendix we discuss the possible mechanism causing the continuous spectra observed in our results. A static approximation of the theory of Stark broadening in plasma shows that the magnitude of the Stark broadening is dependent on the type of optical transition..$^{30}$ By a consideration of the sensitivity of an electronic level to the external field, it is shown that the broadening coefficient is small for the transition between the levels of the same principal quantum number. Since the $345 \mathrm{~nm}$ peak is assigned to $2 s 2{ }^{1} \mathrm{P}^{0}-2 p^{2}{ }^{1} \mathrm{D}$ of the boron ion, this is the case in which very small Stark broadening is expected, while molecular orbitals of $\mathrm{C}_{2}$ may be very sensitive to the external field. However, we still cannot assign the continuous spectra only to the Stark broadening, because our results show that weak continuous spectra still remain after the $\mathrm{C}_{2}$ Swan system or $\mathrm{BO} \alpha$ system has completely disappeared. This is quite clearly seen in the case of graphite in benzene (Fig. 3), boron nitride in benzene (Fig. 7), and boron nitride in $n$-hexane (Fig. 8). Since the Stark broadening is proportional to $N^{2 / 3}$, where $N$ is the number density of perturbing ions or electrons, ${ }^{30}$ decreasing plasma density should cause the band narrowing and the disappearance of the continuous spectra. The disappearance of the line spectra with remaining the continuous spectra, as observed in the present results, means that the line and continuous spectra observed here originated in different emission mechanisms. Furthermore, once an emission band appeared in the spectra, the width of the band remained unchanged with delay time. Since the laser produced plasma is obviously disappearing in this period of time, and hence the plasma density is decreasing, the band narrowing should have occurred in this process, if the Stark broadening had been playing the major role. For these reasons we conclude that the Stark broadening is not the major mechanism giving the continuous spectra, and something else, at least, is present for the strong continuous emission observed in the spectra.

In order to examine if the continuous spectra can be described by the blackbody radiation we compared the spectra with Planck's formula. Qualitatively, Planck's formula predicts that the shorter wavelength side of the spectrum should be steeper than that of the other side, and the cooling of the surface laser-induced plasma should result in a gradual peak shift toward the longer wavelength direction, both of 
which seem to be against the present results. In order to test if Planck's formula fits well to the experimental data, a modified formula,

$$
n(\lambda)=\frac{8 \pi c}{\lambda^{4}} \frac{1}{e^{h c / \lambda k T}-1}
$$

was used, where $n(\lambda)$ is the spectral density of the photon number as a function of wavelength $\lambda, T$ is the temperature of the emitter, $c, h$, and $k$ are the speed of light, Planck constant, and Boltzmann constant, respectively. The reason why the modified formula was used was that the CCD detector does not measure the irradiance in the unit of $\mathrm{W} \mathrm{cm}^{-2}$, but counts the number of the photons entering into each pixel. The best fit curves are plotted by broken lines in Figs. 2-9. It is obvious that at least in the early stage of the emission, Eq. (A1) does not fit well. The large continuous spectra observed in the early stage cannot be described by the blackbody radiation. However, the fit becomes pretty well in the later stage of the emission. In this time range the thermal equilibrium at the target surface was attained and then the emission from the solid surface might have behaved like a blackbody. For the case of boron nitride in $n$-hexane with the delay time of $1000 \mathrm{~ns}$ (Fig. 8), the temperature obtained from the fitting was $3631 \mathrm{~K}$.

When electrons and ions encounter in plasma, it is possible to emit spectrally continuous radiation by the bremsstrahlung or/and radiative recombination process. The former process is characterized by a free final state of the electron while the latter by a bound final state. Although these processes give very different final state of the electron, the emission coefficient due to these two processes can be described by very similar formula for the case of the hydrogenlike atom in a local thermal equilibrium. ${ }^{31,32}$ However, even if a local thermal equilibrium is attained in the present system, the failure in the fitting to Planck's formula means that the optical thickness of the plasma is not enough for Kirchhoff's law to be applied. ${ }^{33}$ Therefore, it is fruitless to discuss the emission spectra in a very quantitative way. Here, therefore, we attempt to make a very rough estimation of the emission coefficient as a function of the number densities of the ions and electrons, and try to clarify whether these processes can explain the continuous spectra observed in the present study.

The simplest process of radiative recombination is the following type;

$$
A^{+}+e \rightarrow A+h \nu,
$$

where $A^{+}, A$, and $e$ denote ion, atom, and electron, respectively. The rate equation of this process should be

$$
\frac{d n_{e}}{d t}=-\alpha n_{e} n^{+}
$$

where $n_{e}$ and $n^{+}$denote the electron and ion density, respectively, and $\alpha$ is the recombination constant. In addition to this simple recombination process, electron emission processes, such as Penning ionization, associative ionization or impact ionization occur in the plasma, and hence, Eq. (A2) seems to be too simplified. Nevertheless, Eq. (A3) is useful for the rough estimation in the present system, because free ions eventually recombine with electron and those processes which occur before the recombination can be effectively included into the phenomenological rate constant $\alpha$. Assuming the absence of multiple charged ions and the local charge neutrality condition, $n_{e}=n^{+}$, the solution of Eq.(A3) becomes

$$
\frac{n_{e}}{n_{0}}=\frac{1}{1+\alpha n_{0} t},
$$

where $n_{0}$ is electron density at $t=0$. The half-life of the free electrons is, therefore, $\left(\alpha n_{0}\right)^{-1}$. The values of $\alpha$ for several ions listed in literature ${ }^{28,35}$ are all in the order of $10^{-12} \mathrm{~cm}^{3} \mathrm{~s}^{-1}$ at $300 \mathrm{~K}$. From the simplest collision picture $\alpha$ is proportional to collision frequency and to collision time, the former is proportional to electron velocity, while the latter is inversely proportional to the electron velocity. This means that $\alpha$ is insensitive to the velocity and also to the temperature in an equilibrium plasma. Actually, in a hydrogen plasma ${ }^{28,35}$ the order of $\alpha$ does not change from $300 \mathrm{~K}$ to $4500 \mathrm{~K}$. The present experiments show that the life time of the continuous emission is in the order of $100 \mathrm{~ns}$. If we assume that the whole continuous emission is due to radiative recombination and that $\alpha$ is $10^{-12} \mathrm{~cm}^{3} \mathrm{~s}^{-1}$, the value of $n_{0}$ is obtained to be $\sim 10^{19} \mathrm{~cm}^{-3}$. This is pretty high compared to that in the gas phase plasma usually observed by the laser ablation. However, the value is still very small compared to the value of atom number density in condensed phases, which is $1.1 \times 10^{23} \mathrm{~cm}^{-3}$ for graphite and 1.0 $\times 10^{23} \mathrm{~cm}^{-3}$ for water. By considering that the plasma is strongly confined between the solid and liquid, and the plume does not expand in contrast to the laser ablation in vacuum, the above electron density obtained by the recombination model seems to be reasonable.

By including the effect of velocity distribution of electrons, the emission intensity for the electron-ion recombination of hydrogenlike ions as a function of light frequency, $\nu$, is given $b^{31}$

$$
\begin{aligned}
& \epsilon_{\nu}=C n_{e} n^{+} \frac{Z^{2}}{T_{e}^{1 / 2}}\left[1-\exp \left(-\frac{h \nu}{k T_{e}}\right)\right], \\
& C=\frac{16 \pi e^{6}}{3 c^{3}\left(6 \pi m_{e}^{3} k\right)^{1 / 2}\left(4 \pi \epsilon_{0}\right)^{3}},
\end{aligned}
$$

where $T_{e}, m_{e}, e$, and $\epsilon_{0}$ are electron temperature, the electron mass, the elementary charge, and the permittivity of the vacuum, respectively, and $Z$ the number of the ion charge. Equation (A6) is valid for the photon energy lower than the ionization energy. Similarly, the emission intensity by the free-free transition or the bremsstrahlung is given by

$$
\epsilon_{\nu}=C n_{e} n^{+} \frac{Z^{2}}{T_{e}{ }^{1 / 2}} \exp \left(-\frac{h \nu}{k T_{e}}\right) .
$$

Equations (A5) and (A7) are closely related, i.e., the only difference is the last factor including the exponential term. For the case of $k T_{e} \ll h \nu$ the emission by the bremsstrahlung is low and the recombination becomes dominant, while for $k T_{e} \gg h \nu$ vice versa. In case that $k T_{e} \sim 1 \mathrm{eV}$ both are in the 
same order in the optical range. In the following we estimate the emission coefficient and compare it with the recombination constant, $\alpha$, discussed above.

The average energy, $P$, emitted per electron per unit time is calculated by integrating Eqs. (A5) and (A7) with frequency and solid angle. ${ }^{33}$ For the free-free transition this integration can be performed analytically. By neglecting multiply charged ions, i.e., $Z=1$ and $n_{e}=n^{+}$, we obtain

$$
P=4 \pi\left(\frac{k}{h}\right) C n_{e} T_{e}{ }^{1 / 2}
$$

The average time required for one electron to lose (3/2)k $k T_{e}$ is

$$
\tau_{\mathrm{rad}}=\frac{(3 / 2) k T_{e}}{P}=\frac{(3 / 2) h T_{e}^{1 / 2}}{4 \pi C n_{e}} .
$$

When $T_{e}$ and $n_{e}$ are measured in units of $\mathrm{K}$ and $\mathrm{cm}^{-3}$, respectively, Eq. (A9) becomes

$$
\tau_{\mathrm{rad}}=1.45 \times 10^{11} \frac{T_{e}^{1 / 2}}{n_{e}} .
$$

Assuming the electron temperature to be $\sim 10^{4} \mathrm{~K}$ and substituting $\tau_{\mathrm{rad}} \approx 10^{-7} \mathrm{~s}$ which is an approximate value obtained from the observed spectra, we get $n_{e} \approx 10^{20} \mathrm{~cm}^{-3}$. This agrees with the aforementioned simple two body recombination process with the electron velocity distribution not taken into account. For the radiative recombination transition such calculation as Eqs. (A8)-(A10) is cumbersome, because the validity of Eq. (A5) is limited to the frequency smaller than that corresponds to the ionization potential. However, if we assume that the integration above the ionization energy is small and that $k T_{e} \approx$ ionization energy, the average energy emitted per electron per unit time due to the recombination process becomes similar to Eq. (A8). This means that the bremsstrahlung and the electron-ion recombination occur in a similar time scale.

In conclusion, the reason we obtain the continuous broad spectra is consistently explained by the electron ion recombination and/or the bremsstrahlung process. On the basis of the above consideration the reason why this spectrum is more prominent in the presence of liquid than solid surfaces in a gaseous atmosphere seems to be straightforward. That is the density of the surface laser-induced plasma is higher for the case of immersing in a liquid, since the expansion of the plasma is restricted by high density of the liquid and that the plasma confined in a small region will result in higher efficiency of the recombination or the bremsstrahlung because of the greater chance for an electron to encounter ions.
${ }^{1}$ J. C. Miller, Laser Ablation: Principles and Applications, edited by J. C. Miller (Springer-Verlag, Berlin, 1994), p. 1.

${ }^{2}$ R. F. Haglund, Jr. and N. Itoh, Laser Ablation: Principles and Applications, edited by J. C. Miller (Springer-Verlag, Berlin, 1994), p. 11.

${ }^{3}$ J. T. Dickinson, S. C. Langford, and L. C. Jensen, Laser Ablation: Mechanisms and Applications, edited by J. C. Miller and R. F. Haglund, Jr. (Springer-Verlag, Berlin, 1991), p. 301.

${ }^{4}$ C. Vivien, J. Hermann, A. Perrone, C. Boulmer-Leborgne, and A. Luches, J. Phys. D 31, 1263 (1998).

${ }^{5}$ Y. F. Lu, Z. M. Ren, W. D. Song, D. S. H. Chan, T. S. Low, K. Gamani, G. Chen, and K. Li, J. Appl. Phys. 84, 2909 (1998).

${ }^{6}$ L. Andrews and T. R. Burkholder, J. Phys. Chem. 95, 8554 (1991).

${ }^{7}$ V. A. Ageev, A. F. Bakhonov, V. V. Zhukovskii, and A. A. Yankovskii, J. Appl. Spectrosc. 64, 683 (1997).

${ }^{8}$ L. Berthe, R. Fabbro, P. Peyre, L. Tollier, and E. Bartnicki, J. Appl. Phys. 82, 2826 (1997).

${ }^{9}$ G. Banas, H. E. Elsayed-Ali, F. V. Lawrence, Jr., and J. M. Rigsbee, J. Appl. Phys. 67, 2380 (1990).

${ }^{10}$ Y. Sano, N. Mukai, K. Okazaki, and M. Obata, Nucl. Instrum. Methods Phys. Res. B 121, 432 (1997).

${ }^{11}$ A. Wakisaka, J. J. Gaumet, Y. Shimizu, Y. Tamori, H. Sato, and K. Tokumaru, J. Chem. Soc., Faraday Trans. 89, 1001 (1993).

${ }^{12}$ M. Nakamura, T. Kitamori, and T. Sawada, J. Phys. Chem. B 103, 1226 (1999).

${ }^{13}$ A. Takami, H. Kurita, and S. Koda, J. Phys. Chem. 99, 875 (1995).

${ }^{14}$ F. Kokai, Jpn. J. Appl. Phys. 36, 3504 (1997).

${ }^{15}$ J. J. Gaumet, A. Wakisaka, Y. Shimizu, and Y. Tamori, J. Chem. Soc., Faraday Trans. 89, 1667 (1993).

${ }^{16}$ G. Koren and J. T. C. Yeh, J. Appl. Phys. 56, 2120 (1984).

${ }^{17}$ Y. Tasaka, M. Tanaka, and S. Usami, Jpn. J. Appl. Phys. 34, 1673 (1995).

${ }^{18}$ S. S. Harilal, R. C. Issac, C. V. Bindhu, V. P. N. Nampoori, and C. P. G. Vallabhan, Jpn. J. Appl. Phys. 36, 134 (1997).

${ }^{19}$ S. S. Harilal, R. C. Issac, C. V. Bindhu, V. P. N. Nampoori, and C. P. G. Vallabhan, J. Appl. Phys. 81, 3637 (1997).

${ }^{20}$ S. S. Harilal, R. C. Issac, C. V. Bindhu, V. P. N. Nampoori, and C. P. G. Vallabhan, J. Appl. Phys. 80, 3561 (1996).

${ }^{21}$ M. Rasanen, J. Y. Liu, T. P. Dzugan, and V. E. Bondybey, Chem. Phys. Lett. 142, 308 (1987).

${ }^{22}$ D. B. Geohegan, Appl. Phys. Lett. 62, 1463 (1993).

${ }^{23}$ P. A. Roland and J. J. Wynne, J. Chem. Phys. 99, 8599 (1993).

${ }^{24}$ R. W. B. Pearse and A. G. Gaydon, The Identification of Molecular Spectra, 4th ed. (Chapman and Hall, London, 1976).

${ }^{25}$ A. R. Striganov and N. S. Sventitskii, Tables of Spectral Lines of Neutral and Ionized Atoms (Plenum, New York, 1968).

${ }^{26}$ G. R. Harrison and F. M. Phelps III, Massachusetts Institute of Technology Wavelength Tables (MIT Press, Cambridge, Massachusetts, 1969).

${ }^{27}$ W. L. Wiese, M. W. Smith, and B. M. Glennon, Atomic Transition Probabilities, National Standard Reference Data Series, National Bureau of Standards 4 (U.S. GPO, Washington, D.C., 1966).

${ }^{28}$ G. Bekefi, C. Deutsch, and B. Yaakobi, Principles of Laser Plasmas, edited by G. Bekefi (Wiley-Interscience, New York, 1976).

${ }^{29}$ H. R. Griem, Plasma Spectroscopy (McGraw-Hill, New York, 1964).

${ }^{30}$ H. R. Griem, Spectral Line Broadening by Plasmas (Academic, London, 1974).

${ }^{31}$ J. Richter, Plasma Diagnostics, edited by W. Llchte-Holtgreven (NorthHolland, Amsterdam, 1968).

${ }^{32}$ H. R. Griem, Principles of Plasma Spectroscopy (Cambridge University Press, Cambridge, 1997).

${ }^{33}$ M. Yamamoto and S. Murayama, Purazuma no Bunkokeisoku (Gakkai Shuppan Senta, Tokyo, 1994) (in Japanese).

${ }^{34}$ W. Demtreoder, Laser Spectroscopy (Springer-Verlag, Berlin, 1982).

${ }^{35}$ R. G. Fowler and W. R. Atkinson, Phys. Rev. 113, 1268 (1959). 